\title{
IMPROVING ROTH'S THEOREM IN THE PRIMES
}

\author{
HARALD ANDRÉS HELFGOTT AND ANNE DE ROTON
}

Abstract. Let $A$ be a subset of the primes. Let

We prove that, if

$$
\delta_{P}(N)=\frac{|\{n \in A: n \leq N\}|}{\mid\{n \text { prime }: n \leq N\} \mid} .
$$

$$
\delta_{P}(N) \geq C \frac{\log \log \log N}{(\log \log N)^{1 / 3}}
$$

for $N \geq N_{0}$, where $C$ and $N_{0}$ are absolute constants, then $A \cap[1, N]$ contains a non-trivial three-term arithmetic progression.

This improves on Green's result Gr], which needs

$$
\delta_{P}(N) \geq C^{\prime} \sqrt{\frac{\log \log \log \log \log N}{\log \log \log \log N}} .
$$

\section{INTRODUCTION}

1.1. History and statement. In 1953, K. Roth [Ro proved that any subset of positive integers of positive density contains infinitely many non trivial three-term arithmetic progressions. More precisely, his result is as follows. Given a set $A \subset \mathbb{Z}^{+}$, we define the density of $A \cap[1, N]$ by $\delta(N)=\frac{1}{N}|\{n \in A: n \leq N\}|$. (We write $|S|$ for the number of elements of a set $S$.) Roth proved that, given any set of integers $A \subset \mathbb{Z}^{+}$such that $\delta(N) \geq C / \log \log N$ for some $N \geq N_{0}$ (where $C$ and $N_{0}$ are absolute constants) there must be at least one non-trivial three-term arithmetic progression in $A \cap[1, N]$. (By a non-trivial arithmetic progression we mean one with non-zero modulus, i.e., $(a, a+d, a+2 d)$ with $d \neq 0$.)

Much later, Heath-Brown HB] (1987) and Szemerédi [Sz (1990) improved this result by showing that it is enough to require that $\delta(N) \geq C(\log N)^{-c}$ for some small positive $c$. By considering Bohr sets where previous arguments had used arithmetic progressions, Bourgain relaxed the condition to $\delta(N) \geq C \sqrt{\log \log N / \log N}$ in [Bo2] (1999) and to $\delta(N) \geq C(\log \log N)^{2}(\log N)^{-2 / 3}$ in [Bo3] (2006).

Van der Corput proved $\mathrm{vdC}$ that the primes contain infinitely many non trivial 3 -term arithmetic progressions. The question then arises - is Roth's theorem true in the primes? That is - must a subset of primes of positive relative density 1 contain a non-trivial 3-term arithmetic progression?

\footnotetext{
${ }^{1}$ Given a subset $A$ of the set $P$ of all primes, we define the relative density $\delta_{P}(N)$ of $A$ to be $\delta_{P}(N)=|\{n \in A: n \leq N\}| / \mid\{n$ prime $: n \leq N\} \mid$. We are asking whether, given $A \subset P$ such that $\delta_{P}(N)>\delta_{0}\left(\delta_{0}>0\right)$ for some sufficiently large $N$, the set $A$ contains a non-trivial 3-term arithmetic progression.
} 
In $\mathrm{Gr}$, B. Green showed that the answer is "yes". He proved that, given any subset $A$ of the primes such that $A \cap[1, N]$ has relative density $\delta_{P}(N) \geq$ $C(\log \log \log \log \log N / \log \log \log \log N)^{1 / 2}$ for some $N \geq N_{0}$, where $C$ and $N_{0}$ are absolute constants, there exists a 3 -term arithmetic progression in $A$.

We prove the following result.

Theorem 1.1. Let $A$ be a subset of the primes. Assume that $A \cap[1, N]$ is of relative density

$$
\delta_{P}(N) \geq C \frac{\log \log \log N}{(\log \log N)^{1 / 3}}
$$

for some $N \geq N_{0}$, where $C$ and $N_{0}$ are absolute constants. Then $A$ contains a non-trivial 3-term arithmetic progression.

In other words, we gain two logs over what was previously known. One of the two logs gained is ultimately due to an enveloping use of a sieve; this idea is by now familiar to the specialists, and, indeed, it will come into our proof via a restriction theorem from [GT] (based partially on work on sieves in [Ra]). The other gain of a log stems from a more essential change in approach.

Our overall procedure is as follows. The first step is to replace the characteristic function $a$ of $A$ by a smoothed-out version $a_{1}$ whose Fourier transform is close to that $a$ (and thus, as can be easily shown, $a_{1}$ behaves like $a$ does when it comes to the number of 3-term progressions). This is much the same as in [Gr, §6]; it is in accord with the general strategy (the "uniformity strategy") described in [Ta, §6]. We then show that the $\ell_{2}$-norm of $a_{1}$ is actually small enough that one can find a set $A^{\prime}$ of large density in the integers such that $a_{1}$ is large on $A^{\prime}$. This reduces the problem over the primes to the problem over the integers.

1.2. Notation. Let $N^{\prime}$ be a positive integer. Let $f: \mathbb{Z} / N^{\prime} \mathbb{Z} \rightarrow \mathbb{C}$ be a function in $l^{1}\left(\mathbb{Z} / N^{\prime} \mathbb{Z}\right)$. We define the Fourier transform of $f$ as the function

$$
\begin{aligned}
& \hat{f}: \mathbb{Z} / N^{\prime} \mathbb{Z} \rightarrow \quad \mathbb{C} \\
& b \mapsto \sum_{n \in \mathbb{Z} / N^{\prime} \mathbb{Z}} f(n) e\left(-n b / N^{\prime}\right),
\end{aligned}
$$

where we write $e(x)$ for $e^{2 \pi i x}$. We write $\pi$ for the reduction map $\pi: \mathbb{Z} \rightarrow \mathbb{Z} / N^{\prime} \mathbb{Z}$. Given $x \in \mathbb{R}$, we define $\{x\}$ to be the distance of $x$ to the nearest integer. We define $\|n\|=\left\{n / N^{\prime}\right\}$; this works because $\{x\}$ depends only on $x \bmod 1$.

Given a finite or countable set $S$, a function $f: S \rightarrow \mathbb{C}$ and a parameter $0<r<$ $\infty$, we define the $\ell_{r}$-norm $|f|_{r}$ of $f$ by $|f|_{r}=\left(\sum_{x \in S}|f(x)|^{r}\right)^{1 / r}$.

1.3. Acknowledgements. Travel was funded by PIMS (Pacific Institute for the Mathematical Sciences) and EPSRC (Engineering and Physical Sciences Research Council). H. A. Helfgott is supported in part by EPSRC grant EP-E054919/1; A. de Roton is partially supported by PIMS and CNRS during the 2009-2010 calendar year. Thanks are due to J. Bourgain, for his prompt reply to some questions, to B. Green, for inviting both authors to visit him at different times, and to I. Laba and T. Wooley, for their constant help. 


\section{From the PRIMES TO THE INTEGERS}

2.1. From the primes to the set $\{n: b+n M$ is prime $\}$. Let us first show that we can focus on the intersection of the primes with an arithmetic progression of large modulus, rather than work on all the primes.

Lemma 2.1. Let $\alpha, z$ be positive real numbers and $N$ be a large integer. We define $M=\prod_{p \leq z}$ p. Let $A$ be a subset of the primes less than $N$ such that $|A| \geq \alpha N / \log N$. Then there exists some arithmetic progression $P(b)=\{b+n M: 1 \leq n \leq N / M\}$ such that

$$
|P(b) \cap A| \gg \alpha \frac{\log z}{\log N} \frac{N}{M}-\log z,
$$

where the implied constant is absolute.

Proof. If $(b, M) \neq 1$, the set $\{m \in P(b): m$ prime $\}$ is empty. Since the progressions $P(b)$ with $(b, M)=1$ are distinct, we have

$$
\sum_{b:(b, M)=1}|A \cap P(b)|=|A|-|A \cap[1, M-1]| \geq \alpha \frac{N}{\log N}-M .
$$

But $|\{b \leq M:(b, M)=1\}| \sim M / \log z \sim e^{z} / \log z$. Therefore there exists some progression $P(b)$ such that

$$
|A \cap P(b)| \gg\left(\alpha \frac{N}{\log N}-M\right) \frac{\log z}{M} \gg \alpha \frac{\log z}{\log N} \frac{N}{M}-\log z .
$$

The passage to an arithmetic progression $b+n M$ of large modulus is exactly what Green and Tao [GT2, p. 484] call the "W-trick" (due to Green's use of the letter $W$ for $M$ in $[\mathrm{Gr}]$ ). Green uses the fact that such a passage removes all but the largest peaks in the Fourier transform of the primes, whereas we simply use in a more direct way the fact that the elements of $\{n: b+n M$ prime $\}$ are not forbidden from having small divisors. Of course, these are two manifestations of the same idea.

Now, we fix $z=\frac{1}{3} \log N, M=\prod_{p<z} p$, and let $N^{\prime}$ be the least prime larger than $\lceil 2 N / M\rceil$. (The requirement $N^{\prime}>\lceil 2 N / M\rceil$ will ensure that no new three-term arithmetic progressions are created when we apply the reduction map $\pi: \mathbb{Z} \rightarrow \mathbb{Z} / N^{\prime} \mathbb{Z}$ to a set contained in $[1, N / M]$.) By Bertrand's postulate, $N^{\prime} \ll N / M$. Let $A$ be a subset of the primes less than $N$ such that $|A| \geq \alpha N / \log N$. We assume $\alpha \geq(\log N) N^{-1 / 2}$ (say) and obtain from Lemma 2.1 that there is an arithmetic progression $P(b)$ such that $|P(b) \cap A| \gg \alpha(\log z / \log N) N^{\prime}$. We define $A_{0}$ to be

$$
A_{0}=\pi\left(\left\{n=\frac{m-b}{M}: m \in P(b) \cap A\right\}\right) .
$$

This is a subset of $\pi\left(\left\{n \in\left[1, N^{\prime}\right]: b+n M\right.\right.$ is prime $\left.\}\right)$ satisfying

$$
\left|A_{0}\right| \gg \alpha \frac{\log z}{\log N} N^{\prime}
$$


Our task is to show that there is a non-trivial three-term arithmetic progression in $A_{0} \subset \mathbb{Z} / N^{\prime} \mathbb{Z}$. It will follow immediately that there is a non-trivial three-term arithmetic progression in $A \subset \mathbb{Z}$.

2.2. From the set $\{n: b+n M$ is prime $\}$ to the set of integers. Let $a$ be the normalised characteristic function of $A_{0}$, i.e., $a=\left(\log N /\left(N^{\prime} \log z\right)\right) \mathbf{1}_{A_{0}}$. Fixing $\delta>0$ and $\varepsilon \in(0,1 / 4)$ to be chosen later, define $R:=\left\{x \in \mathbb{Z} / N^{\prime} \mathbb{Z}:|\hat{a}(x)| \geq \delta\right\} \cup\{1\}$ and the Bohr set

$$
B:=\left\{n \in \mathbb{Z} / N^{\prime} \mathbb{Z}: \forall x \in R,\|n x\| \leq \varepsilon\right\}
$$

We also define on $\mathbb{Z} / N^{\prime} \mathbb{Z}$ the functions $\sigma=\frac{1}{|B|} \mathbf{1}_{B}$ and $a_{1}=a * \sigma$.

To begin with, we remark that $|a|_{1}=\left(\log N /\left(N^{\prime} \log z\right)\right)\left|A_{0}\right| \gg \alpha$ and $\left|a_{1}\right|_{1}=$ $|a|_{1}|\sigma|_{1}=|a|_{1}$. Thus $\left|a_{1}\right|_{1} \gg \alpha$, i.e., $a_{1}$ is large in $\ell_{1}$-norm. We will later show that $a_{1}$ is small in $\ell_{2}$-norm. These bounds on the $\ell_{1}$-norm and the $\ell_{2}$-norm will enable us to find a large set of integers on which $a_{1}$ is $\gg \frac{1}{N}$. This will enable us to reduce the problem for large subsets of the primes to Roth's theorem for large subsets of the integers.

We first have to show that $a_{1}$ is "close" to $a$ in the sense that we care about, namely - we must show that $a_{1}$ is large on all three terms of many three-term arithmetic progressions if and only if the same is true of $a$ (i.e., if and only if $A$ contains many three-term arithmetic progressions). More precisely, our aim is to bound from above the quantity

$$
\Delta=N^{\prime} \cdot\left|\sum_{n_{1}, n_{2}, n_{3} \text { in AP }} a\left(n_{1}\right) a\left(n_{2}\right) a\left(n_{3}\right)-\sum_{n_{1}, n_{2}, n_{3} \text { in } \mathrm{AP}} a_{1}\left(n_{1}\right) a_{1}\left(n_{2}\right) a_{1}\left(n_{3}\right)\right|
$$

where the sums $\sum_{n_{1}, n_{2}, n_{3}}$ in AP are over all triples $\left(n_{1}, n_{2}, n_{3}\right)$ of elements of $\mathbb{Z} / N^{\prime} \mathbb{Z}$ in arithmetic progression. Since $\left(n_{1}, n_{2}, n_{3}\right)$ is an arithmetic progression if and only if $n_{1}+n_{3}=2 n_{2}$,

$$
\Delta=\left|\sum_{m} \hat{a}(-2 m)(\hat{a}(m))^{2}-\sum_{m} \hat{a}_{1}(-2 m)\left(\hat{a}_{1}(m)\right)^{2}\right|
$$

as we can see simply by replacing all Fourier transforms by their definitions and using the fact that $\sum_{m} e\left(\left(n_{1}+n_{3}-2 n_{2}\right) m / N^{\prime}\right)=0$ when $n_{1}+n_{3}-2 n_{2} \neq 0$.

We will show that $\Delta$ is small, namely, $\Delta \ll \epsilon+\delta$. First note that, since $a_{1}=a * \sigma$ and so $\hat{a_{1}}=\hat{a} \hat{\sigma}$,

$$
\Delta \leq \sum_{m}\left|\hat{a}(-2 m)-(\hat{a}(m))^{2}\right|\left|1-\hat{\sigma}(-2 m)(\hat{\sigma}(m))^{2}\right|
$$


For $x \in R$, since $\sigma$ is supported on $B$ and $\sum_{n} \sigma(n)=1$, we have

$$
\begin{aligned}
|\hat{\sigma}(x)-1| & =\left|\sum_{n \in \mathbb{Z} / N^{\prime} \mathbb{Z}} \sigma(n) e\left(n x / N^{\prime}\right)-1\right| \\
& =\left|\sum_{n \in \mathbb{Z} / N^{\prime} \mathbb{Z}} \sigma(n)-1+\sum_{n \in \mathbb{Z} / N^{\prime} \mathbb{Z}} \sigma(n)\left(e\left(n x / N^{\prime}\right)-1\right)\right| \\
& \leq \sum_{n \in B} \sigma(n)\left|e\left(n x / N^{\prime}\right)-1\right| \ll \sum_{n \in B} \sigma(n)\|n x\| \ll \varepsilon .
\end{aligned}
$$

Similarly, for $x \in R$,

$$
|\hat{\sigma}(-2 x)-1| \ll \sum_{n \in B} \sigma(n)\|-2 n x\| \ll \sum_{n \in B} \sigma(n) \varepsilon=\varepsilon
$$

and so

$$
\left|1-\hat{\sigma}(-2 x) \hat{\sigma}(x)^{2}\right| \ll \varepsilon
$$

for $x \in R$, i.e., when $|\hat{a}(x)| \geq \delta$.

Before we proceed further, we need to bound $\hat{a}$ in an average sense.

Lemma 2.2. For $p>2$,

$$
\sum_{m \in \mathbb{Z} / N^{\prime} \mathbb{Z}}|\hat{a}(m)|^{p} \ll_{p} 1 .
$$

This is the same as [Gr, Lemma 6.6]; the only difference is that our function $a$ was defined with a much larger modulus $M$ than in $[\mathrm{Gr}$, and thus we must use a restriction theorem for an upper-bound sieve, rather than a restriction theorem for the primes (such as [Bo, (4.39)]).

Proof. Applying [GT, Prop. 4.2] with $F(n)=b+n M$ and $R=N^{\prime 1 / 10}$, we obtain that, for $p>2$ and any complex sequence $\left(b_{n}\right)_{n}$,

$$
\sum_{m \in \mathbb{Z} / N^{\prime} \mathbb{Z}}\left|\frac{1}{N^{\prime}} \sum_{n=1}^{N^{\prime}} b_{n} \beta(n) e\left(-m n / N^{\prime}\right)\right|^{p} \ll_{p}\left(\frac{1}{N^{\prime}} \sum_{n=1}^{N^{\prime}}\left|b_{n}\right|^{2} \beta(n)\right)^{p / 2},
$$

where $\beta$ is an enveloping sieve function with $R=N^{\prime 1 / 10}$. This means that, according to [GT, Prop. 3.1], $\beta: \mathbb{Z}^{+} \rightarrow \mathbb{R}$ is a non-negative function satisfying the majorant property

with

$$
\beta(n) \gg \mathfrak{S}_{F}^{-1} \cdot \log R \cdot \mathbf{1}_{X_{R !}}(n)
$$

$$
\begin{gathered}
\mathfrak{S}_{F}=\prod_{p} \frac{\gamma(p)}{1-1 / p}, \\
\gamma(p)=\frac{1}{p}|\{n \in \mathbb{Z} / p \mathbb{Z},(p, b+n M)=1\}|= \begin{cases}(1-1 / p) & \text { if } p>z \\
1 & \text { if } p \leq z\end{cases}
\end{gathered}
$$


and

$$
X_{R !}=\{n \in \mathbb{Z}: \forall d \leq R(b+n M, d)=1\} .
$$

In particular, for any integer $n \in A_{0}$, we have $n \in X_{R}$ ! and

$$
\beta(n) \gg(\log R) \prod_{p \leq z}(1-1 / p)^{-1} \gg \frac{\log N}{\log z}
$$

We apply (2.5) to the sequence $\left(b_{n}\right)_{n}$ defined by

$$
b_{n}= \begin{cases}\frac{1}{\beta(n)} a(n) & \text { if } n \in A_{0} \\ 0 & \text { otherwise }\end{cases}
$$

and get

$$
\begin{aligned}
\sum_{m \in \mathbb{Z} / N^{\prime} \mathbb{Z}}|\hat{a}(m)|^{p} & =\left(N^{\prime}\right)^{p} \sum_{m \in \mathbb{Z} / N^{\prime} \mathbb{Z}}\left|\frac{1}{N^{\prime}} \sum_{n \in \mathbb{Z} / N^{\prime} \mathbb{Z}} a(n) e(-m n / N)\right|^{p} \\
& \ll_{p}\left(N^{\prime}\right)^{p / 2}\left(\sum_{n \in \mathbb{Z} / N^{\prime} \mathbb{Z}} \frac{1}{\beta(n)} a(n)^{2}\right)^{p / 2} \\
& \ll_{p}\left(N^{\prime}\right)^{p / 2}\left(\sum_{n \in \mathbb{Z} / N^{\prime} \mathbb{Z}} \frac{\log z}{\log N}\left(\frac{\log N}{N^{\prime} \log z}\right) a(n)\right)^{p / 2} \\
& \ll_{p}\left(\sum_{n \in \mathbb{Z} / N^{\prime} \mathbb{Z}} a(n)\right)^{p / 2} \ll_{p} 1,
\end{aligned}
$$

since $a(n)$ was normalised so that $\sum_{n} a(n) \ll 1$.

By Hölder's inequality and Lemma 2.2, we have

$$
\sum_{m \in \mathbb{Z} / N^{\prime} \mathbb{Z}}\left|\hat{a}(-2 m) \hat{a}(m)^{2}\right| \leq\left(\sum_{m \in \mathbb{Z} / N^{\prime} \mathbb{Z}}|\hat{a}(m)|^{5 / 2}\right)^{2 / 5}\left(\sum_{m \in \mathbb{Z} / N^{\prime} \mathbb{Z}}|\hat{a}(m)|^{10 / 3}\right)^{3 / 5} \ll 1 .
$$

Hence, by (2.3),

$$
\sum_{m:|\hat{a}(m)| \geq \delta}\left|\hat{a}(-2 m) \hat{a}(m)^{2}\right|\left|1-\hat{\sigma}(-2 m) \hat{\sigma}(m)^{2}\right| \ll \varepsilon
$$


On the other hand (again by Hölder, and again by Lemma 2.2),

$$
\begin{aligned}
\sum_{m:|\hat{a}(m)|<\delta}\left|\hat{a}(-2 m) \hat{a}(m)^{2}\right|\left|1-\hat{\sigma}(-2 m) \hat{\sigma}(m)^{2}\right| \leq 2 \sum_{m:|\hat{a}(m)|<\delta}\left|\hat{a}(-2 m) \hat{a}(m)^{2}\right| \\
\quad \leq 2\left(\sum_{m:|\hat{a}(m)|<\delta}|\hat{a}(m)|^{5 / 2}\right)^{2 / 5}\left(\sum_{m:|\hat{a}(m)|<\delta}|\hat{a}(m)|^{10 / 3}\right)^{3 / 5} \\
\quad \leq 2\left(\sum_{m \in \mathbb{Z} / N^{\prime} \mathbb{Z}}|\hat{a}(m)|^{5 / 2}\right)^{2 / 5}\left(\delta^{5 / 3} \sum_{m \in \mathbb{Z} / N^{\prime} \mathbb{Z}}|\hat{a}(m)|^{5 / 3}\right)^{3 / 5} \ll \delta .
\end{aligned}
$$

Thus

$$
\Delta \ll(\varepsilon+\delta) .
$$

2.3. An upper bound for the $\ell_{2}$-norm of $a_{1}$. Our aim in this subsection is to bound from above the $\ell_{2}$-norm of of the function $a_{1}=a * \sigma$. (This will later enable us to show that $a_{1}$ is in some sense close to the characteristic function of a set of large density in the integers.) We will prove that $\left|a_{1}\right|_{2} \ll 1 / \sqrt{N^{\prime}}$, where the implied constant is absolute.

Recall that we write $\pi$ for the reduction map $\pi: \mathbb{Z} \rightarrow \mathbb{Z} / N^{\prime} \mathbb{Z}$. Given a function $f: \mathbb{Z} / N^{\prime} \mathbb{Z} \rightarrow \mathbb{C}$, we can lift it to a function $\tilde{f}: \mathbb{Z} \rightarrow \mathbb{C}$ supported on the interval $\left[-\left(N^{\prime}-1\right) / 2,\left(N^{\prime}-1\right) / 2\right]$ :

$$
\tilde{f}(n)= \begin{cases}f\left(n \bmod N^{\prime}\right) & \text { if } n \in\left[-\frac{N^{\prime}-1}{2}, \frac{N^{\prime}-1}{2}\right], \\ 0 & \text { otherwise. }\end{cases}
$$

By the definition of $A_{0}$ and $a$, we see that $A_{0} \subset \pi\left(\left[1,\left(N^{\prime}-1\right) / 2\right]\right)$, and thus $a$ is supported on $\pi\left(\left[1,\left(N^{\prime}-1\right) / 2\right]\right)$. By the definition of $R, B$ and $\sigma$ and the assumption $\varepsilon<1 / 4$, we see that $\sigma$ is supported on $\pi\left(\left[-N^{\prime} / 4, N^{\prime} / 4\right]\right)$. Thus $|a * \sigma|_{2}=|\tilde{a} * \tilde{\sigma}|_{2}$.

By the definition of $a$, we have $0 \leq \tilde{a}(n) \leq \lambda(n)$, where $\lambda: \mathbb{Z} \rightarrow \mathbb{R}$ is defined by

$$
\lambda(n)= \begin{cases}\frac{\log N}{N^{\prime} \log z} & \text { if } 1 \leq n \leq N^{\prime} \text { and } b+n M \text { is prime } \\ 0 & \text { otherwise. }\end{cases}
$$

Recall that $\sigma$ is non-negative, and thus $\tilde{\sigma}$ is non-negative. Hence $|\tilde{a} * \tilde{\sigma}|_{2} \leq|\lambda * \tilde{\sigma}|_{2}$. We conclude that

$$
\left|a_{1}\right|_{2}=|a * \sigma|_{2}=|\tilde{a} * \tilde{\sigma}|_{2} \leq|\lambda * \tilde{\sigma}|_{2} .
$$

It is thus our task to prove that $|\lambda * \tilde{\sigma}|_{2} \ll 1 / \sqrt{N^{\prime}}$.

We proceed as follows:

$$
\begin{aligned}
\sum_{n}|\tilde{\sigma} * \lambda(n)|^{2} & =\sum_{n}\left|\sum_{m} \tilde{\sigma}(m) \lambda(n-m)\right|^{2} \\
& =\sum_{m_{1}} \sum_{m_{2}} \tilde{\sigma}\left(m_{1}\right) \tilde{\sigma}\left(m_{2}\right) \sum_{n} \lambda\left(n+m_{1}\right) \lambda\left(n+m_{2}\right),
\end{aligned}
$$

where we recall that $\sigma(m)=\sigma(-m)$ (by the definition of $B$ and $\sigma$ ). 
Lemma 2.3. Let $\lambda$ be as in 2.7). Then, for any integers $m_{1}, m_{2}$,

$$
\sum_{n} \lambda\left(n+m_{1}\right) \lambda\left(n+m_{2}\right) \ll\left\{\begin{array}{cll}
\log N /\left(N^{\prime} \log z\right) & \text { if } & m_{1}=m_{2}, \\
\frac{1}{N^{\prime}} \prod_{p \mid\left(m_{1}-m_{2}\right), p>z} \frac{p}{p-1} & \text { if } & m_{1} \neq m_{2},
\end{array}\right.
$$

where the implied constant is absolute.

Proof. The case $m_{1}=m_{2}$ follows from Brun-Titchmarsh:

$$
\begin{aligned}
\sum_{n} \lambda^{2}(n+m) & =\left(\frac{\log N}{N^{\prime} \log z}\right)^{2} \mid\left\{m \leq n \leq N^{\prime}+m: b+(n-m) M \text { is prime }\right\} \mid \\
& \ll\left(\frac{\log N}{N^{\prime} \log z}\right)^{2} \frac{N^{\prime} M}{\varphi(M) \log N^{\prime}} \\
& \ll\left(\frac{\log N}{N^{\prime} \log z}\right)^{2} \frac{N^{\prime}}{\log N^{\prime}} \prod_{p \mid M}(1-1 / p)^{-1} \\
& \ll \frac{\log N}{N^{\prime} \log z} .
\end{aligned}
$$

To obtain the case $m_{1} \neq m_{2}$, we will use a result based on Selberg's sieve. (This is a familiar type of application of upper-bound sieves, similar to the proof that the number of twin primes up to $N$ is at most a constant times its conjectured value.) It is clear that $\sum_{n} \lambda\left(n+m_{1}\right) \lambda\left(n+m_{2}\right)$ equals $\left(\log N /\left(N^{\prime} \log z\right)\right)^{2}$ times

$$
\mid\left\{1 \leq n \leq N^{\prime}: b+n M \text { and } b+\left(n+m_{2}-m_{1}\right) M \text { are primes }\right\} \mid .
$$

By [HR, Thm. 5.7],

$$
(2.10) \ll \prod_{p}\left(1-\frac{\rho(p)-1}{p-1}\right)\left(1-\frac{1}{p}\right)^{-1} \frac{N^{\prime}}{\left(\log N^{\prime}\right)^{2}},
$$

where the implied constant is absolute. (We are implicitly using the fact that $\log M \ll \log N^{\prime}$, and thus the term in the third line of [HR, (8.3)] is $=1+o(1)$.) Here $\rho(p)$ is the number of solutions $x \in \mathbb{Z} / p \mathbb{Z}$ to

$$
(b+x M)\left(b+\left(x+m_{2}-m_{1}\right) M\right) \equiv 0 \bmod p
$$

for $p$ prime. It is easy to see that $\rho(p)=0$ if $p \mid M$ (i.e., if $p \leq z), \rho(p)=1$ if $p>z$ and $p \mid\left(m_{2}-m_{1}\right)$, and $\rho(p)=2$ if $p>z$ and $p \nmid\left(m_{2}-m_{1}\right)$. Hence

$$
\begin{aligned}
(2.10) & \ll \prod_{p \leq z}\left(1-\frac{1}{p}\right)^{-2} \prod_{\substack{p>z \\
p \mid m_{1}-m_{2}}}\left(1-\frac{1}{p}\right)^{-1} \frac{N^{\prime}}{\left(\log N^{\prime}\right)^{2}} \\
& \ll \prod_{\substack{p>z \\
p \mid m_{1}-m_{2}}} \frac{p}{p-1} \frac{N^{\prime}(\log z)^{2}}{\left(\log N^{\prime}\right)^{2}} .
\end{aligned}
$$

The statement follows. 
Let us now evaluate the last line of (2.8), with Lemma 2.3 in hand. The contribution of the diagonal terms $\left(m_{1}=m_{2}\right)$ in (2.8) is $\ll \log N /\left(|B| N^{\prime} \log z\right)$. The contribution of the non-diagonal terms $\left(m_{1} \neq m_{2}\right)$ is

$$
\ll \frac{1}{N^{\prime}} \sum_{\substack{m_{1} \\ m_{2} \neq m_{1}}} \sum_{\substack{m_{2} \\ \sigma}} \tilde{\sigma}\left(m_{1}\right) \tilde{\sigma}\left(m_{2}\right) \prod_{\substack{p>z \\ p \mid m_{1}-m_{2}}} \frac{p}{p-1} .
$$

Recall that $\tilde{\sigma}$ is supported on $\left[-N^{\prime} / 4, N^{\prime} / 4\right]$, and thus $\left|m_{2}-m_{1}\right| \leq N^{\prime} / 2<N^{\prime}$ whenever $\tilde{\sigma}\left(m_{1}\right) \tilde{\sigma}\left(m_{2}\right) \neq 0$.

Now, a non-zero integer $m$ with $|m| \leq N^{\prime}$ cannot have more than $\log N^{\prime} / \log z$ prime factors $p>z$. Since $x \mapsto x /(x-1)$ is decreasing on $x$, this means that

$$
\prod_{\substack{p>z \\ p \mid m}} \frac{p}{p-1} \leq\left(\frac{z}{z-1}\right)^{\left(\log N^{\prime}\right) /(\log z)} .
$$

Now $(z /(z-1))^{z} \ll 1$ (because $\lim _{n}(1+1 / n)^{n}=e$ ) and

$$
\frac{\log N^{\prime}}{\log z} \ll \frac{\log N}{\log \log N}<\log N \ll z .
$$

Hence

$$
\prod_{\substack{p>z \\ p \mid m}} \frac{p}{p-1} \ll 1
$$

for any $m \neq 0$ with $|m| \leq N^{\prime}$. Thus

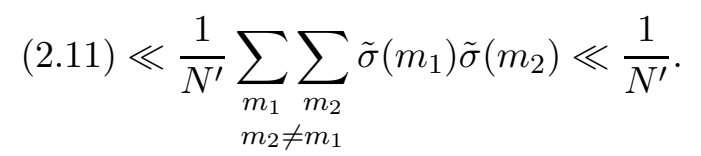

Putting everything together, we conclude that

$$
\sum_{n}|\tilde{\sigma} * \lambda(n)|^{2} \ll \frac{1}{N^{\prime}}\left(\frac{\log N}{|B| \log z}+1\right) .
$$

The right side is $\ll 1 / N^{\prime}$ as long as $|B| \gg \log N / \log z$.

Now, as is well-known (see, e.g., [TV, Lem. 4.20]),

$$
|B| \gg \varepsilon^{r} N^{\prime}
$$

where $r=|R|$. (The proof of this is a simple pigeonhole argument.) Since by (2.4) we have $\sum_{m}|\hat{a}(m)|^{5 / 2} \ll 1$, we know that that the set of $x \in \mathbb{Z} / N^{\prime} \mathbb{Z}$ with $|\hat{a}(x)| \geq \delta$ has at most $\ll \delta^{-5 / 2}$ elements. Thus, $r \ll \delta^{-5 / 2}$.

Hence all that we need for $|B| \geq \log N / \log z$ to hold is that $\varepsilon^{\delta^{-5 / 2}} \geq N^{-1 / 2}$ (say). In other words, we need $|\log \varepsilon| \cdot \delta^{-5 / 2} \leq \frac{1}{2} \log N$. We will recall that we need to satisfy this condition at the end. 
2.4. Extracting a dense set from $a_{1}$. We now have a function $a_{1}: \mathbb{Z} / N^{\prime} \mathbb{Z} \rightarrow \mathbb{R}_{0}^{+}$ of $\ell_{2}$ norm $\ll 1 / \sqrt{N^{\prime}}$. Its $\ell_{1}$ norm is $\gg \alpha$, where $\alpha$ is the density of our original set $A$ on the primes. We must show that there is a large set on which $a_{1}$ is large.

Lemma 2.4. Let $S$ be a set with $N^{\prime}$ elements. Let $a: S \rightarrow \mathbb{R}_{0}^{+}$and $0<\alpha<1$ be such that

(a) $\|a\|_{1} \geq \alpha$;

(b) $\|a\|_{2}^{2} \leq c / N^{\prime}$.

Then there exists a subset $A^{\prime}$ of $S$ such that

(a) $\left|A^{\prime}\right| \geq \alpha^{2} N^{\prime} /(4 c)$;

(b) $\forall n \in A^{\prime}, a(n) \geq \alpha / 2 N^{\prime}$.

Proof. If $A^{\prime}=\left\{n: a(n) \geq \alpha /\left(2 N^{\prime}\right)\right\}$, then

$$
\begin{aligned}
\alpha & \leq \sum_{n} a(n) \leq \frac{\alpha}{2 N^{\prime}}\left(N^{\prime}-\left|A^{\prime}\right|\right)+\sum_{n \in A^{\prime}} a(n) \\
& \leq \frac{\alpha}{2 N^{\prime}}\left(N^{\prime}-\left|A^{\prime}\right|\right)+\sqrt{\left|A^{\prime}\right|} \sqrt{\frac{c}{N^{\prime}}}
\end{aligned}
$$

by $\|a\|_{2} \leq c / N^{\prime}$ and Cauchy's inequality. In other words, $f\left(\sqrt{\left|A^{\prime}\right|}\right) \leq 0$, where $f(x)=\frac{\alpha}{2 N^{\prime}}\left(x^{2}-2 \frac{\sqrt{c N^{\prime}}}{\alpha} x+N^{\prime}\right)$. Completing the square, we see that $f(x) \leq 0$ implies $x \geq \frac{\sqrt{c N^{\prime}}}{\alpha}-\sqrt{\left(\frac{c}{\alpha^{2}}-1\right) N^{\prime}}$. Hence

$$
\left|A^{\prime}\right| \geq N^{\prime} \cdot\left(\frac{\sqrt{c}}{\alpha}-\sqrt{\frac{c}{\alpha^{2}}-1}\right)^{2} \geq \frac{\alpha^{2} N^{\prime}}{4 c} .
$$

We apply Lemma 2 to $a_{1}$ with the bound $\left\|a_{1}\right\|_{2}^{2} \leq c / N^{\prime}$ being provided by our work in $\$ 2.3$. We get a subset $A^{\prime}$ of $\mathbb{Z} / N^{\prime} \mathbb{Z}$ such that $\left|A^{\prime}\right| \geq \alpha^{2} N^{\prime} /(4 c) \gg \alpha^{2} N^{\prime}$ and $a_{1}(n) \geq \alpha /\left(2 N^{\prime}\right)$ for every $n \in A^{\prime}$. Hence

$$
\sum_{m, d} a_{1}(m) a_{1}(m+d) a_{1}(m+2 d) \geq \frac{\alpha^{3} Z}{8 N^{\prime 3}},
$$

where $Z$ is the number of 3 -term arithmetic progressions in $A^{\prime}$.

Lemma 2.5. Let $A^{\prime} \subset \mathbb{Z} / N^{\prime} \mathbb{Z}$, where $N^{\prime}$ is a prime. Assume $\left|A^{\prime}\right| \geq \eta N^{\prime}, \eta>0$. The number of 3-term arithmetic progressions in $A^{\prime}$ is then at least

$$
\frac{\eta N^{\prime 2}}{c_{0} \exp \left(c_{1} \eta^{-3 / 2}(\log (1 / \eta))^{3}\right)}
$$

where $c_{0}$ and $c_{1}$ are absolute constants.

Proof. We will proceed much as in [Gr, Lem. 6.8]; the basic argument goes back to Varnavides [Va]. Bourgain's best result on three-term arithmetic progressions in the integers [Bo3, Thm. 1] states that, for given $L$ and $\eta \gg(\log \log L)^{2}(\log L)^{-2 / 3}$, every 
subset of $\{1,2, \ldots, L\}$ with $\geq \eta L$ elements contains at least one non-trivial threeterm arithmetic progression. This can be rephrased as follows: there are constants $c_{0}$ and $c_{1}$ such that, if $L \geq c_{0} \exp \left(c_{1} \eta^{-3 / 2}(\log (1 / \eta))^{3}\right)$, then any subset of $\{1, \cdots, L\}$ of density at least $\eta / 2$ contains a non-trivial three-term arithmetic progression. (Here we are simply expressing $L$ in terms of the density, rather than the density in terms of $L$.)

It follows that, given an arithmetic progression $S_{a, d}=\{a+d, a+2 d, a+3 d, \ldots, a+$ $L d\}$ in $\mathbb{Z} / N^{\prime} \mathbb{Z}\left(a, d \in \mathbb{Z} / N^{\prime} \mathbb{Z}, d \neq 0, L \leq N^{\prime}\right)$ whose intersection with $A^{\prime}$ has at least $(\eta / 2) L$ elements, there is at least one non-trivial three-term arithmetic progression in $A^{\prime} \cap S \subset \mathbb{Z} / N^{\prime} \mathbb{Z}$. (Note that there is no need for the progression $S$ to be the reduction $\bmod N^{\prime}$ of a progression in the integers $\left\{1,2, \ldots, N^{\prime}\right\}$; the argument works regardless of this.) If we consider all arithmetic progressions of length $L$ and given modulus $d \neq 0$ in $\mathbb{Z} / N^{\prime} \mathbb{Z}$, we see that each element of $A^{\prime}$ is contained in exactly $L$ of them. Hence, $\sum_{a}\left|S_{a, d} \cap A^{\prime}\right|=L\left|A^{\prime}\right| \geq \eta N^{\prime} L$, and so (for $d \neq 0$ fixed) $\left|S_{a, d} \cap A^{\prime}\right| \geq(\eta / 2) L$ for at least $(\eta / 2) N^{\prime}$ values of $a$. Varying $d$, we get that $\left|S_{a, d} \cap A^{\prime}\right| \geq(\eta / 2) L$ for at least $(\eta / 2) N^{\prime}\left(N^{\prime}-1\right)$ arithmetic progressions $S_{a, d}$. By the above, each such intersection $S_{a, d} \cap A^{\prime}$ contains at least one non-trivial three-term arithmetic progression.

Each non-trivial three-term arithmetic progression $a_{1}, a_{2}, a_{3}$ in $\mathbb{Z} / N^{\prime} \mathbb{Z}$ can be contained in at most $L(L-1)$ arithmetic progressions $\{a+d, a+2 d, \ldots, a+L d\}$ of length $L$ (the indices of $a_{1}$ and $a_{2}$ in the progression of length $L$ determine the progression). Hence, when we count the three-term arithmetic progressions coming from the intersections $S_{a, d} \cap A^{\prime}$, we are counting each such progression at most $L(L-1)$ times. Thus we have shown that $A^{\prime}$ contains at least

$$
\frac{\eta}{2} \frac{N^{\prime}\left(N^{\prime}-1\right)}{L(L-1)} \geq \frac{\eta}{2} \frac{N^{\prime 2}}{L^{2}}
$$

distinct non-trivial three-term arithmetic progressions for

$$
L=\left\lceil c_{0} \exp \left(c_{1} \eta^{-3 / 2}(\log (1 / \delta))^{3}\right)\right\rceil,
$$

provided that $L \leq N^{\prime}$. If $L>N^{\prime}$, the bound in the statement of the lemma is trivially true (as there is always at least one trivial three-term arithmetic progression in $A^{\prime}$.)

From (2.12) and Lemma 2.5, we conclude that

$$
\begin{aligned}
\sum_{m, d} a_{1}(m) a_{1}(m+d) a_{1}(m+2 d) & \geq \frac{\alpha^{3}}{8 N^{\prime 3}} \frac{\alpha^{2}}{8 c} \frac{\left(N^{\prime}\right)^{2}}{c_{0} \exp \left(c_{1}\left(\alpha^{2} / 4 c\right)^{-3 / 2}\left(\log \left(4 c / \alpha^{2}\right)\right)^{3}\right)} \\
& \geq \frac{1}{N^{\prime}} \frac{1}{c_{2} \exp \left(c_{3} \alpha^{-3}(\log (1 / \alpha))^{3}\right)},
\end{aligned}
$$

where $c_{2}, c_{3}>0$ are absolute constants.

\section{Conclusion}

Assume that $A$ contains no non-trivial three-term arithmetic progressions. Then $A_{0}$ (defined in (2.1) ) contains no non-trivial three term arithmetic progressions, and 
SO

$$
\sum_{m, d} a(m) a(m+d) a(m+2 d)=\sum_{m, d} a(m)^{3} \ll\left(\frac{\log N}{N^{\prime} \log z}\right)^{2} .
$$

We also have

$$
\Delta=N^{\prime}\left|\sum_{m, d} a(m) a(m+d) a(m+2 d)-\sum_{m, d} a_{1}(m) a_{1}(m+d)_{1}(m+2 d)\right| \ll(\varepsilon+\delta),
$$

by the definition (2.2) and (2.6). Lastly, we have just shown that

$$
\sum_{m, d} a_{1}(m) a_{1}(m+d) a_{1}(m+2 d) \geq \frac{1}{N^{\prime}} \frac{1}{c_{2} \exp \left(c_{3} \alpha^{-3}(\log (1 / \alpha))^{3}\right)}
$$

(see (2.13)). We conclude that

$$
\frac{1}{c_{2} \exp \left(c_{3} \alpha^{-3}(\log (1 / \alpha))^{3}\right)} \ll \varepsilon+\delta+\frac{1}{N^{\prime}}\left(\frac{\log N}{\log z}\right)^{2} .
$$

Recall that $z=(\log N) / 3$. There are constants $c_{4}, c_{5}$ such that, for

$$
\delta=\varepsilon=\frac{1}{c_{4}} \exp \left(-c_{5} \alpha^{-3} \log ^{3}(1 / \alpha)\right),
$$

we get a contradiction with (3.1), provided that $N$ is larger than an absolute constant and $\alpha \geq(\log N)^{-1 / 4}$, say. These values of $\delta$ and $\varepsilon$ satisfy $|\log \varepsilon| \delta^{-2.5} \leq(\log N) / 2$ as long as

$$
\left(\log \left(c_{4}\right)+c_{5} \alpha^{-3} \log ^{3}(1 / \alpha)\right) \cdot c_{4}^{2.5} \exp \left(2.5 c_{5} \alpha^{-3} \log ^{3}(1 / \alpha)\right) \leq \frac{1}{2} \log N .
$$

Therefore we have a contradiction if $\alpha \geq C \log \log \log N(\log \log N)^{-1 / 3}$, where $C$ is a large enough constant and $N$ is larger than an absolute constant. Theorem 1.1 is thereby proven.

\section{REFERENCES}

[Bo] Bourgain, J., On $\Lambda(p)$-subsets of squares, Israel J. Math. 67 (1989), no. 3, 291-311.

[Bo2] Bourgain, J., On triples in arithmetic progression, Geom. Funct. Anal. 9 (1999), no. 5, 968-984.

[Bo3] Bourgain, J., Roth's theorem on progressions revisited,

[Gr] Green, B., Roth's theorem in the primes, Ann. of Math. (2) 161 (2005), 1609-1636.

[GT] Green, B., and T. Tao, Restriction theory of the Selberg sieve, with applications, Journal de théorie des nombres de Bordeaux 18 (2006), no. 1, p. 147-182.

[GT2] Green, B., and T. Tao, The primes contain arbitrarily long arithmetic progressions, Ann. of Math. (2) 167 (2008), no. 2, 481-547.

[HR] Halberstam, H., and H. E. Richert, Sieve methods, Academic Press, 1974.

[HB] Heath-Brown, D. R., Integer sets containing no arithmetic progressions, J. London Math. Soc. (2) 35 (1987), no. 3, 385-394.

[Ra] Ramaré, O., On Snirel'man's constant, Ann. Scu. Norm. Pisa 22 (1995), 645-706.

[Ro] Roth, K. F., On certain sets of integers, J. London Math. Soc. 28 (1953), 104-109.

[Sz] Szemerédi, E., Integer sets containing no arithmetic progressions, Acta Math. Hungar. 56 (1990), no. 1-2, 155-158.

[Ta] Tao, T., Arithmetic progressions and the primes, Collect. Math. 2006, Vol. Extra, 37-88. 
[TV] Tao, T., and V. Vu, Additive combinatorics, Cambridge University Press, 2006.

[vdC] van der Corput, J. G., Über Summen von Primzahlen und Primzahlquadraten, Math. Ann. 116 (1939), 1-50.

[Va] Varnavides, P., On certain sets of positive density, J. London Math. Soc. 34 (1959) 358-360.

H. A. Helfgott, Department of Mathematics, University of Bristol, Bristol BS8 1TW, UNITED KingDOM

E-mail address: h.andres.helfgott@bristol.ac.uk

A. De Roton, Institut Elie Cartan, UMR 7502, Nancy-Université, CNRS, INRIA, B.P. 239, 54506 Vandouevre-lès-Nancy Cedex, France; A. De Roton, Pacific Institute For the Mathematical Sciences (CNRS UMI PIMS) University of British Comlumbia VAnCouver BC V6T 1Z2 CANADA

E-mail address: deroton@iecn.u-nancy.fr 